\title{
The BRST-invariant vacuum state of the Gribov-Zwanziger theory
}

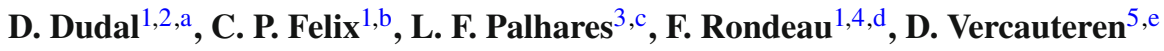 \\ ${ }^{1}$ Department of Physics, KU Leuven Campus Kulak Kortrijk, Etienne Sabbelaan 53 bus 7657, 8500 Kortrijk, Belgium \\ ${ }^{2}$ Department of Physics and Astronomy, Ghent University, Krijgslaan 281-S9, 9000 Ghent, Belgium \\ ${ }^{3}$ Departamento de Física Teórica, UERJ, Universidade do Estado do Rio de Janeiro, Rua São Francisco Xavier 524, Maracanã, Rio de Janeiro \\ 20550-013, Brazil \\ ${ }^{4}$ Ecole Normale Supérieure Paris-Saclay, Avenue du Président Wilson 61, 94235 Cachan Cedex, France \\ ${ }^{5}$ Institute of Research and Development, Duy Tân University, P809, 3 Quang Trung, Hải Châu, Da Nang, Vietnam
}

Received: 7 February 2019 / Accepted: 20 August 2019 / Published online: 31 August 2019

(C) The Author(s) 2019

\begin{abstract}
We revisit the effective action of the GribovZwanziger theory, taking into due account the BRST symmetry and renormalization (group invariance) of the construction. We compute at one loop the effective potential, showing the emergence of BRST-invariant dimension 2 condensates stabilizing the vacuum. This paper sets the stage at zero temperature, and clears the way to studying the GribovZwanziger gap equations, and particularly the horizon condition, at finite temperature in future work.
\end{abstract}

\section{Introduction}

Up until now, quark and gluon confinement has not been rigorously proven. It is well known that the perturbative formalism fails for non-Abelian gauge theories at low energy, since the coupling constant $g^{2}$ is strong. To get reliable results in the infrared (IR) in the continuum formulation, non-perturbative methods are needed. For a small selection of such methods and obtained results, let us refer for example to [1-15]. Notice that the continuum formulation requires gauge fixing, in which case lattice analogues of dedicated gauge fixings can be a powerful ally giving complementary insights, see [16-23] for some relevant works in this area.

Motivated by this, a number of studies over the past decade have focused on the gluon, quark and also ghost propagator in the infrared region, where color degrees of freedom are confined. Although these objects are unphysical by themselves

\footnotetext{
a e-mail: david.dudal@kuleuven.be

b e-mail: caroline.felix@kuleuven.be

ce-mail: leticia.palhares@uerj.br

d e-mail: francois.rondeau@ens-paris-saclay.fr

e e-mail: vercauterendavid@duytan.edu.vn
}

- being gauge variant - they are nevertheless the basic building blocks, next to the interaction vertices, entering gaugeinvariant objects directly linked to physically relevant quantities such as the spectrum, decay constants, critical exponents and temperatures, etc. One of the most striking features that all these non-perturbative approaches share is that the gluon attains a dynamical mass scale, thereby resolving its massless pole. Notice that this gluon mass scale has nothing to do with an observable massive gluon, as the gluon is not part of the spectrum. Indeed, its spectral properties are not compatible with an asymptotic $S$-matrix observable. This also means that establishing unitarity at the level of strongly interacting gluons is a meaningless question, rather one should prove the non-perturbative unitarity at the level of gluon and quark bound states (glueballs, hadrons and possibly hybrids). To the best of our knowledge, this is an open question which would basically amount to prove confinement and the Yang-Mills mass gap, and certainly not the topic of this paper. Direct model-independent lattice evidence for the unphysical spectral nature of the gluon came from its non-positive Schwinger function (temporal correlator), e.g. [16,24], non-positive spectral function, e.g. [25-28], or even possible occurrence of complex conjugate mass poles, [29]. Such features also appear in aforementioned analytical approaches, see our extensive reference list.

One particular way to deal with non-perturbative physics at the level of elementary degrees of freedom is by dealing with the Gribov issue $[9,30]$ : the fact that there is no unique way of selecting one representative configuration of a given gauge orbit in covariant gauges [31]. As there is also no rigourous way to deal properly with the existence of gauge copy modes in the path integral quantization procedure, in this paper we will use a well-tested formalism available to 
deal with the issue, which is known as the Gribov-Zwanziger (GZ) formalism: a restriction of the path integral to a smaller subdomain of gauge fields $[30,32,33]$.

This approach was first proposed for the Landau and the Coulomb gauges . It long suffered from a serious drawback: its concrete implementation seemed to be inconsistent with BRST (Becchi-Rouet-Stora-Tyutin [34-36]) invariance of the gauge-fixed theory, which clouded its interpretation as a gauge (fixed) theory. Only more recently was it realized by some of us and colleagues how to overcome this complication to get a BRST-invariant restriction of the gauge path integral . As a bonus, the method also allowed the generalization of the GZ approach to the linear covariant gauges, amongst others [37-40].

Another issue with the original GZ approach was that some of its major leading-order predictions did not match the corresponding lattice output. Indeed, in the case of the Landau gauge, the GZ formalism by itself predicts at tree level a gluon propagator vanishing at momentum $p=0$, next to, more importantly, a ghost propagator with a stronger than $1 / p^{2}$ singularity for $p \rightarrow 0$. Although the latter fitted well in the Kugo-Ojima confinement criterion [41], it was at odds with large volume lattice simulations $[17,18]$. By now, several analytical takes exist on this, all being compatible, qualitatively and/or quantitatively, with lattice data, not only for elementary propagators but also for vertices. In the GZ formalism, in particular, the situation can be remedied by correctly incorporating the effects of certain mass dimension two condensates, the importance of which was already stressed before in papers like [42-46]. This idea was first put on the table in $[3,4]$ and later on a self-consistent computational scheme was constructed in [47] based on the effective action formalism for local composite operators developed in $[45,48]$, the renormalization of which was proven in [49]. Unfortunately, the explicit computation of the effective action was not achieved at the time, while the setup was still based on the BRST-breaking GZ proposal.

The goal of this paper is thus to revisit, in the newly established BRST-invariant setting, the dynamical generation of $d=2$ condensates, the latter themselves affiliated to BRSTinvariant operators. Said otherwise, we will explicitly construct the non-perturbative GZ vacuum, which will be shown to have a lower vacuum energy compared to the original GZ action. Moreover, we show that the original action represents a totally unstable point of the effective potential, while the formation of the condensates properly produces a minimum. The GZ vacuum thus stabilizes itself by the formation of non-trivial condensates, which in return affect the dynamics of the field excitations above that vacuum. The practical problems to compute the effective potential that plagued [47] are circumvented here by a clever use of Hubbard-Stratonovich transformations.
This paper is organized as follows. In Sect. 2, we briefly introduce the BRST-invariant Gribov-Zwanziger formalism for the class of linear covariant gauges. The transition from the Gribov-Zwanziger to the Refined Gribov-Zwanziger (RGZ) procedure is described in Sect. 3. We also concisely explain the renormalization group equation aspects of the effective action construction for a set of $d=2$ BRST invariant local composite operators in Sect. 4. In the Sect. 5, the one-loop calculation of the effective potential is presented. Finally, in Sect. 6, the physical solution is identified in $\overline{\mathrm{MS}}$ and in general schemes.

\section{The BRST-invariant Gribov-Zwanziger action in linear covariant gauges}

It is well known that at low energy, we have to deal with Gribov copies, in principle both with "large" and infinitesimal ones. In the low-energy regime, such copies are not suppressed because the coupling constant $g$ is large [30]. A way to avoid the ambiguity - or at least the ambiguity coming from the infinitesimal ones - is to restrict the functional integral over the gauge fields to a specific region $\Omega$ in field space where no infinitesimal Gribov copies exist - as was originally proposed by Gribov in the Landau gauge [30]. As the Gribov ambiguity exists for any covariant gauge [31], it will in particular be present in the class of widely used linear covariant gauges, to which Feynman gauge and Landau gauge belong. It was only recently discussed how to treat these copies in linear covariant gauges other than the Landau gauge $[37-40,50]$.

The construction eliminating (infinitesimal) Gribov copies in general linear covariant gauges is based on the field $A_{\mu}^{h}$, which is the gauge transformed configuration of $A_{\mu}$ minimizing the functional

$$
\begin{aligned}
& f_{A}[u] \equiv \min _{\{u\}} \operatorname{Tr} \int d^{d} x A_{\mu}^{u} A_{\mu}^{u}, \\
& A_{\mu}^{u}=u^{\dagger} A_{\mu} u+\frac{i}{g} u^{\dagger} \partial_{\mu} u,
\end{aligned}
$$

which is obtained through iterative minimization of the functional $f_{A}[u]$ along the gauge orbit of $A_{\mu}$ [51-53]. The field $A_{\mu}^{h}$ is a non-local power series in the gauge field; iterative minimization produces the following local minimum:

$$
\begin{aligned}
A_{\mu}^{h}= & \left(\delta_{\mu \nu}-\frac{\partial_{\mu} \partial_{\nu}}{\partial^{2}}\right) \phi_{\nu}, \quad \partial_{\mu} A_{\mu}^{h}=0, \\
\phi_{\nu}= & A_{\nu}-i g\left[\frac{1}{\partial^{2}} \partial A, A_{\nu}\right] \\
& +\frac{i g}{2}\left[\frac{1}{\partial^{2}} \partial A, \partial_{\nu} \frac{1}{\partial^{2}} \partial A\right]+\mathcal{O}\left(A^{3}\right) .
\end{aligned}
$$


It is worth pointing out that the quantity $A_{\mu}^{h}$ is gauge invariant order by order [37-40,50]. If we couple $A_{\mu}^{h}$ to the Yang-Mills action in a general linear covariant gauge, it seems this will result in a non-local quantum field theory. Fortunately, the field $A_{\mu}^{h}$ can be localized by adding an auxiliary Stueckelberg field $\xi^{a}[37-40,50,54]$ so that

$A_{\mu}^{h}=\left(A^{h}\right)_{\mu}^{a} T^{a}=h^{\dagger} A_{\mu}^{a} T^{a} h+\frac{i}{g} h^{\dagger} \partial_{\mu} h, \quad h=\mathrm{e}^{i g \xi^{a} T^{a}}$,

and by imposing that $A_{\mu}^{h}$ is transverse, $\partial_{\mu} A_{\mu}^{h}=0$. Now, the local gauge invariance of $A_{\mu}^{h}$ under a gauge transformation $u \in S U(N)$ can be appreciated from

$h \rightarrow u^{\dagger} h, \quad h^{\dagger} \rightarrow h^{\dagger} u, \quad A_{\mu} \rightarrow u^{\dagger} A_{\mu} u+\frac{i}{g} u^{\dagger} \partial_{\mu} u$

Using this field $A_{\mu}^{h}$, a Gribov region $\Omega$ not containing any infinitesimal Gribov copies is given by

$\Omega=\left\{A_{\mu}^{a} ; \partial_{\mu} A_{\mu}^{a}=i \alpha b^{a}, \quad \mathcal{M}^{a b}\left(A^{h}\right)=-\partial_{\mu} D_{\mu}^{a b}\left(A^{h}\right)>0\right\}$,

where a Hermitian Faddeev-Popov-like operator, ${ }^{1} \mathcal{M}^{a b}\left(A^{h}\right)$ $=-\delta^{a b} \partial^{2}+g f^{a b c}\left(A^{h}\right)_{\mu}^{c} \partial_{\mu}$, is required to be positive. Notice that we have to assume here that every gauge orbit passes through this region $\Omega$. In fact, to our knowledge, it is not even formally proven that every gauge configuration can be transformed into the linear covariant gauge to begin with. This has only been firmly established for the Landau gauge [55].

Implementing the positivity of the Hermitian operator $-\partial D\left(A^{h}\right)$ is a sufficient condition to kill off a large set of gauge copies in linear covariant gauges, namely those that are continuously connected to infinitesimal copies in Landau gauge, as has been discussed in [37]. More precisely, we impose that the Fourier transform of the inverse operator of $-\partial D\left(A^{h}\right)$ displays no poles for $p^{2}>0$. This constraint can, in the thermodynamic limit, be lifted into the path integral using a saddle point evaluation. The saddle point equation is nothing else than the horizon condition, which in its original, non-local, form reads in $d$ dimensions

$$
\begin{aligned}
\langle h(x)\rangle= & d\left(N^{2}-1\right), \\
h(x)= & g^{2} \gamma^{4} \int d^{d} x f^{a k c} A_{\mu}^{h, k}(x) \\
& \times\left[-\partial_{\mu} D_{\mu}^{a b}\left(A^{h}\right)\right]_{(x, y)}^{-1} f^{b m c} A_{\mu}^{h, m}(y)
\end{aligned}
$$

We refer to $[37,38]$ for the detailed derivation, see also [9, $30,32,33,56]$.

\footnotetext{
1 This is not the Faddeev-Popov operator for a generic linear covariant gauge, the latter is given by the non-Hermitian operator $-\partial D(A)$.
}

The total action implementing the Gribov horizon condition in a general linear covariant gauge is given by

$S=S_{Y M}+S_{G F}+S_{G Z}+S_{\varepsilon}$.

In this expression, $S_{Y M}$ is the Yang-Mills action

$S_{Y M}=\frac{1}{4} \int d^{d} x F_{\mu \nu}^{a} F_{\mu \nu}^{a} ;$

$S_{G F}$ denotes the Faddeev-Popov gauge fixing in the linear covariant gauge:

$S_{G F}=\int d^{d} x\left(\frac{\alpha_{g}}{2} b^{a} b^{a}+i b^{a} \partial_{\mu} A_{\mu}^{a}+\bar{c}^{a} \partial_{\mu} D_{\mu}^{a b}(A) c^{b}\right)$,

with $\alpha_{g}$ the gauge parameter, which is zero for the Landau gauge; and $S_{G Z}$ is the Gribov-Zwanziger action in its local form, which can be written as

$$
\begin{aligned}
S_{G Z}= & \int d^{d} x\left[\bar{\varphi}_{\mu}^{a c} \partial_{\nu} D_{\nu}^{a b}\left(A^{h}\right) \varphi_{\mu}^{b c}-\bar{\omega}_{\mu}^{a c} \partial_{\nu}\left(D_{\nu}^{a b}\left(A^{h}\right) \omega_{\mu}^{b c}\right)\right] \\
& -\gamma^{2} g \int d^{4} x\left[f^{a b c}\left(A^{h}\right)_{\mu}^{a}\left(\varphi_{\mu}^{b c}+\bar{\varphi}_{\mu}^{b c}\right)\right. \\
& \left.+\frac{d}{g}\left(N^{2}-1\right) \gamma^{2}\right],
\end{aligned}
$$

The localizing fields $\left(\bar{\varphi}_{\mu}^{a c}, \varphi_{\mu}^{a c}\right)$ are a pair of complexconjugate bosonic fields, while $\left(\bar{\omega}_{\mu}^{a c}, \omega_{\mu}^{a c}\right)$ a pair of anticommuting complex-conjugate fields. The fields $\bar{\eta}^{a}$ and $\eta^{a}$ are also ghost-like, while $\gamma$ is the Gribov parameter, which is dynamically fixed by a gap equation $[32,33,38,56]$,

$\left\langle f^{a b c}\left(A^{h}\right)_{\mu}^{a}\left(\varphi_{\mu}^{b c}+\bar{\varphi}_{\mu}^{b c}\right)\right\rangle=2 d\left(N^{2}-1\right) \frac{\gamma^{2}}{g^{2}}$,

also known as the horizon condition. This equation can be succinctly rewritten as

$\frac{\partial \Gamma}{\partial \gamma^{2}}=0$

where $\Gamma$ is the quantum action defined by

$\mathrm{e}^{-\Gamma}=\int[\mathcal{D} \Phi] \mathrm{e}^{-S}$

with $[\mathcal{D} \Phi]$ the Haar measure of integration over all the quantum fields present in the action. By integrating over the auxiliary fields in $(7 d)$, the equivalence of the local with the non-local version can be easily established, including the fact that the horizon condition (9) is equivalent to the saddle point constraint (6).

Finally, the term $S_{\varepsilon}$

$S_{\varepsilon}=\int d^{d} x\left(\varepsilon^{a} \partial_{\mu}\left(A^{h}\right)_{\mu}^{a}+\bar{\eta}^{a} \partial_{\mu} D_{\mu}^{a b}\left(A^{h}\right) \eta^{b}\right)$

implements, through the Lagrange multiplier $\varepsilon$, the transversality of the composite operator $\left(A^{h}\right)_{\mu}^{a}$, namely $\partial_{\mu}\left(A^{h}\right)_{\mu}^{a}=$ 
0 . The fields $\bar{\eta}^{a}$ and $\eta^{a}$ are also ghost-like and account for the Jacobian accompanying the constraint.

The action $S$ in Eq. (7a) enjoys an exact BRST invariance, $s S=0$ and $s^{2}=0$, expressed by $[37-40,50]$

$$
\begin{aligned}
& s A_{\mu}^{a}=-D_{\mu}^{a b} c^{b}, \\
& s c^{a}=\frac{g}{2} f^{a b c} c^{b} c^{c}, \quad s \bar{c}^{a}=i b^{a}, \\
& s b^{a}=0, \\
& s \varphi_{\mu}^{a b}=0, \quad s \omega_{\mu}^{a b}=0, \\
& s \bar{\omega}_{\mu}^{a b}=0, \quad s \bar{\varphi}_{\mu}^{a b}=0, \\
& s \varepsilon^{a}=0, \quad s\left(A^{h}\right)_{\mu}^{a}=0, \\
& s h^{i j}=-i g c^{a}\left(T^{a}\right)^{i k} h^{k j} .
\end{aligned}
$$

Notice that the gap equation (8) is a BRST-invariant condition. The multiplicative renormalizability of this construction was proven, to all orders, in $[57,58]$.

\section{Refined Gribov-Zwanziger action}

In [3], it was noticed that the GZ formalism in Landau gauge is plagued by non-perturbative dynamical instabilities, leading to the formation of $d=2$ condensates like $\left\langle A_{\mu}^{a} A_{\mu}^{a}\right\rangle$ and $\left\langle\bar{\varphi}_{\mu}^{a b} \varphi_{\mu}^{a b}-\bar{\omega}_{\mu}^{a b} \omega_{\mu}^{a b}\right\rangle$, which are energetically favored $[3,4,47]$. Later, similar features were noticed in the Maximal Abelian gauge GZ formulation [59,60]. This led to the Refined Gribov-Zwanziger formalism, which explicitly takes into account the effects of these condensates.

In this paper, we will work out in detail the dynamical RGZ formalism in linear covariant gauges. In order to do so, we will couple the BRST-invariant operators $A_{\mu}^{h, a} A_{\mu}^{h, a}$ and $\bar{\varphi}_{\mu}^{a b} \varphi_{\mu}^{a b}$ to the GZ action via the local composite operator (LCO) formalism. As a final result, the Refined GribovZwanziger (RGZ) action (27) will be obtained. With this RGZ action, the dominant IR ghost behavior is $1 / p^{2}$, while the gluon propagator, at tree-level but in the new improved vacuum, is given by

$$
\begin{aligned}
& \left\langle A_{\mu}^{a}(p) A_{\nu}^{b}(-p)\right\rangle \\
& =\frac{p^{2}+M^{2}}{p^{4}+\left(M^{2}+m^{2}\right) p^{2}+M^{2} m^{2}+\lambda^{4}} \mathcal{P}_{\mu \nu}(p) \delta^{a b} \\
& \quad+\frac{\alpha_{g}}{p^{2}} L_{\mu \nu} \delta^{a b},
\end{aligned}
$$

where

$\mathcal{P}_{\mu \nu}(p)=\delta_{\mu \nu}-\frac{p_{\mu} p_{v}}{p^{2}}, \quad L_{\mu \nu}=\frac{p_{\mu} p_{v}}{p^{2}}$,

are the transversal and longitudinal projectors, $\lambda^{4}=2 g^{2} N \gamma^{4}$, and $M^{2}$ and $m^{2}$ are the mass scales linked to the condensates $\left\langle\bar{\varphi}_{\mu}^{a b} \varphi_{\mu}^{a b}\right\rangle$ and $\left\langle A_{\mu}^{h, a} A_{\mu}^{h, a}\right\rangle$, respectively (see later). It can be shown, [39], that the longitudinal form factor remains bare, as is usual in the linear covariant gauge. This fact is also confirmed non-perturbatively using lattice simulations [23,61] and is consistent with the findings in $[62,63]$ as well.

For later usage, we remind here that, using the Nielsen identities, it can be shown that the poles of the gluon propagator are gauge parameter and renormalization scale independent order per order, even in the GZ case. See the detailed discussion in [40]. Evidently, BRST invariance is crucial here as this underlies the Nielsen identities. We will later on use this knowledge.

Depending on the relative size of the mass scales appearing in (13), the propagator can develop complex-conjugate poles. If (13) is fitted to lattice data, the complex pole scenario is clearly preferred [64-66]. These complex poles evidently remove the gluon from the physical spectrum, which could offer an intuitive explanation of why gluons are unobservable. Notice that these complex poles also occur explicitly in other approaches, see $[14,29,67]$.

To compute the effective potential of the above-mentioned condensates, we add the local sources $\tau$ and $Q$, coupled to the relevant local composite operators, to the action $S$ given in (7a):

$\Sigma=S+S_{A^{2}}+S_{\varphi \bar{\varphi}}+S_{\mathrm{vac}}$.

In this expression, we have, including the $Z$-factors in the conventions of [47], that

$$
\begin{aligned}
S_{A^{2}} & =\int d^{d} x Z_{A}\left(Z_{\tau \tau} \tau+Z_{\tau Q} Q\right) \frac{1}{2} A_{\mu}^{h, a} A_{\mu}^{h, a}, \\
S_{\bar{\varphi} \varphi} & =\int d^{d} x Z_{Q Q} Z_{\varphi} Q \bar{\varphi}_{\mu}^{a c} \varphi_{\mu}^{a c}, \\
S_{\mathrm{vac}} & =-\int d^{d} x\left(\frac{Z_{\zeta} \zeta}{2} \tau^{2}+Z_{\alpha} \alpha Q^{2}+Z_{\chi} \chi Q \tau\right) .
\end{aligned}
$$

In the above expressions, we already used the fact that the source $Q$ has no mixing with $\tau$ (i.e. $Z_{Q \tau}=0$ ), while $\tau$ does mix with $Q$, see later. At the operator level, this means $\bar{\varphi} \varphi$ mixes with $A^{h} A^{h}$, while $A^{h} A^{h}$ renormalizes on its own. The sources are BRST singlets,

$s \tau=0, \quad s Q=0$.

When computing the generating functional, new divergences proportional to $\tau^{2}, Q^{2}$ and $\tau Q$ appear. This happens because of the divergences appearing in correlation functions such as $\left\langle\mathcal{O}_{j}(x) \mathcal{O}_{j}(y)\right\rangle$, with $\mathcal{O}_{i}$ one of the $d=2$ operators added to the RGZ action. This is why the term $S_{\text {vac }}$ given in (15d) is necessary. The counterterms, which come with new and a priori free parameters $\alpha, \chi$ and $\zeta$ (so-called LCO parameters), will absorb the divergences in $\tau^{2}, Q^{2}$ and $Q \tau$, i.e. via $\delta \zeta \tau^{2}, \delta \alpha Q^{2}$ and $\delta \chi Q \tau$. We will momentarily discuss how to fix the (finite) parameters themselves, while maintaining full multiplicative renormalizability. This method was 
originally developed in [48], see also [45,68]. The generalization, including operator mixing, was worked out first in [47], and we will rely on the latter reference.

Given that the main purpose of this work is to compute $d=2$ vacuum condensates which are BRST invariant, we can actually make use of the full power of BRST. Indeed, we can choose an appropriate gauge for explicit computation. Clearly, the Landau gauge is singled out, as in that case $A^{h} A^{h}$ collapses into $A^{2}$. Loosely speaking, this is clear from expression (2). A more formal proof based on integrating over the auxiliary fields $\xi, \epsilon, \eta$ and $\bar{\eta}$ is provided in [40], establishing that the BRST invariant action for $\alpha_{g} \rightarrow 0$ exactly reduces to that of the original GZ action in Landau gauge (modulo the extra $d=2$ operators of course).

As such, we can rely on the algebraic renormalization analysis already performed in [47], establishing the renormalizability of the action to all orders of perturbation theory. Moreover, it was shown that the sources $(Q, \tau)$ have the following renormalization structure

$$
\left(\begin{array}{c}
Q_{0} \\
\tau_{0}
\end{array}\right)=\left(\begin{array}{cc}
Z_{Q Q} & 0 \\
Z_{\tau Q} & Z_{\tau \tau}
\end{array}\right)\left(\begin{array}{c}
Q \\
\tau
\end{array}\right)
$$

\section{Essential points of the LCO formalism}

This section is largely based on [47, Sect. 4.1]. In order to make the paper self-contained, we now review the main steps.

We are interested in the generating functional

$$
e^{-\Gamma(J)}=\int[\mathcal{D} \Phi] e^{-\Sigma}
$$

where $J=\left(\begin{array}{l}Q \\ \tau\end{array}\right)$ and the classical action, with sources, has been written down in (15a). At the bare level and in dimensional regularization $(d=4-\epsilon)$, we have

$$
\begin{aligned}
- & \frac{1}{2} \zeta_{0} \tau_{0}^{2}-\alpha_{0} Q_{0}^{2}-\chi_{0} Q_{0} \tau_{0} \\
= & -\mu^{-\epsilon}\left(\frac{1}{2} \zeta \tau^{2}+\alpha Q^{2}+\chi Q \tau\right. \\
& \left.+\frac{1}{2} \delta \zeta \tau^{2}+\delta \alpha Q^{2}+\delta \chi Q \tau\right),
\end{aligned}
$$

where we used $\delta \zeta, \delta \alpha$ and $\delta \chi$ to denote the corresponding vacuum counterterms [47], necessary to remove the divergences in the sources squared that arise when computing the generating functional. We also already introduced the renormalization scale $\mu$ necessary for dimensional reasons.

The renormalization matrix can be translated into an anomalous dimension matrix $\gamma$ [47],

$$
\gamma=\left(\begin{array}{cc}
Z_{Q Q}^{-1} \mu \frac{\partial}{\partial \mu} Z_{Q Q} & 0 \\
-Z_{\tau Q} \mu \frac{\partial}{\partial \mu} Z_{Q Q}+Z_{\tau \tau}^{-1} \mu \frac{\partial}{\partial \mu} Z_{\tau Q} & Z_{\tau \tau}^{-1} \mu \frac{\partial}{\partial \mu} Z_{\tau \tau}
\end{array}\right)
$$

$$
=\left(\begin{array}{cc}
\gamma_{Q Q} & 0 \\
\gamma_{21} & \gamma_{\tau \tau}
\end{array}\right)
$$

so that

$\mu \frac{\partial}{\partial \mu} J=-\gamma \cdot J$.

Next, deriving (19) w.r.t. $\mu$ and identifying terms in $Q^{2}, \tau^{2}$ and $Q \tau$, we find 3 coupled differential equations

$$
\begin{aligned}
& \beta\left(g^{2}\right) \frac{\partial}{\partial g^{2}} \frac{\zeta\left(g^{2}\right)}{2}=\frac{\epsilon}{2} \delta \zeta-\frac{1}{2} \beta\left(g^{2}\right) \frac{\partial}{\partial g^{2}}(\delta \zeta) \\
& \quad+\gamma_{\tau \tau}\left(g^{2}\right)(\zeta+\delta \zeta), \\
& \beta\left(g^{2}\right) \frac{\partial}{\partial g^{2}} \alpha\left(g^{2}\right)=\epsilon \delta \alpha-\beta\left(g^{2}\right) \frac{\partial}{\partial g^{2}}(\delta \alpha) \\
& \quad+2 \gamma_{Q Q}\left(g^{2}\right)(\alpha+\delta \alpha)+\Gamma_{21}\left(g^{2}\right)(\chi+\delta \chi), \\
& \beta\left(g^{2}\right) \frac{\partial}{\partial g^{2}} \chi\left(g^{2}\right)=\epsilon \delta \chi-\beta\left(g^{2}\right) \frac{\partial}{\partial g^{2}}(\delta \chi) \\
& \quad+\gamma_{Q Q}\left(g^{2}\right)(\chi+\delta \chi)+\gamma_{\tau \tau}\left(g^{2}\right)(\chi+\delta \chi) \\
& \quad+\Gamma_{21}\left(g^{2}\right)(\zeta+\delta \zeta) .
\end{aligned}
$$

where, following the LCO formalism [48], we made $\zeta\left(g^{2}\right)$, $\alpha\left(g^{2}\right)$ and $\xi\left(g^{2}\right)$ functions of $g^{2}$, such that they are no longer free parameters but completely determinable by solving the previous renormalization-group based equations. In practice, this happens order per order in perturbation theory by using a Laurent expansion in $g^{2}$. This choice (which is unique, see $[48,68])$ is compatible with multiplicative renormalizability of the parameters, in addition to ensuring a homogeneous renormalization group equation of the standard type for the generating functional,

$$
\left(\mu \frac{\partial}{\partial \mu}+\beta\left(g^{2}\right) \frac{\partial}{\partial g^{2}}+\int d^{d} x J \cdot \gamma \cdot \frac{\delta}{\delta J}\right) \Gamma(J)=0 .
$$

Note that in deriving the relations (22), the finiteness of $(\zeta, \alpha, \xi)$ plays a role.

\section{Computation of the effective action}

Notice that the action $\Sigma$ in (15a) has three terms quadratic in the sources. These terms introduce a conceptual difficulty: the interpretation of the effective action $\Gamma$ as an energy density. Indeed, when the sources $J$ are linearly coupled to fields $\sigma$, the functional $\Gamma(J)$ can be Legendre transformed into $\Gamma(\sigma)$. However, if $\Gamma(J)$ contains squares (or higher powers) of the sources, these terms would not cancel out in the Legendre transform, such that the interpretation of $\Gamma$ as an energy density is unclear.

In $[45,48]$, it was shown how to circumvent this apparent problem by a suitable Hubbard-Stratonovich transformation. In the case of mixing sources/operators, a generalization of 
this strategy was first worked out in [47]. Here, we will use a slightly different version from that of [47], which offers the advantage that - despite the observations in $[45,48]-$ it is not necessary to perform $(n+1)$-loop computations to get $n$-loop results with the LCO formalism. That this is possible was first noticed in [69].

To get rid of these quadratic terms in the sources, we proceed by introducing two auxiliary fields $\sigma_{1}$ and $\sigma_{2}$ through two identities

$$
\begin{aligned}
& 1=\int\left[\mathcal{D} \sigma_{1}\right] e^{-\frac{1}{2 Z_{\zeta}} \int d^{d} x\left(\sigma_{1}+\frac{\bar{a}}{2} A^{2}+\bar{b} Q+\bar{c} \tau\right)^{2}}, \\
& 1=\int\left[\mathcal{D} \sigma_{2}\right] e^{+\frac{1}{2 Z_{\alpha}} \int d^{d} x\left(\sigma_{2}+\bar{d} \bar{\varphi} \varphi+\bar{e} Q+\frac{\bar{f}}{2} A^{2}\right)^{2}},
\end{aligned}
$$

with which we multiply the integral in (18). The positive sign in the exponent of the second integral (24b) obviously makes it infinite. However, we should remind that all functional integrals are actually defined only up to an infinite constant, often not explicitly written. Actually, what we are doing by inserting this "infinite identity" can be seen as a rescaling of the infinite constant hidden in expression (18). Doing this rescaling in a subtle way, a careful choice of the coefficients $\bar{a}$ to $\bar{f}$ allows us to eliminate all the quadratic terms in sources appearing in the partition function.

A straightforward computation shows that we have to choose the coefficients

$$
\begin{aligned}
& \bar{a}=\frac{Z_{A} Z_{\tau \tau}}{\sqrt{\zeta}} \mu^{\epsilon / 2} \\
& \bar{b}=-\frac{Z_{\chi} \chi}{\sqrt{\zeta}} \mu^{-\epsilon / 2}, \\
& \bar{c}=-Z_{\zeta} \sqrt{\zeta} \mu^{-\epsilon / 2}, \\
& \bar{d}=\frac{Z_{\varphi} Z_{Q Q}}{\sqrt{-2 \alpha+\frac{Z_{\chi}^{2} \chi^{2}}{Z_{\alpha} Z_{\zeta} \zeta}} \mu^{\epsilon / 2},} \\
& \bar{e}=Z_{\alpha} \sqrt{-2 \alpha+\frac{Z_{\chi}^{2} \chi^{2}}{Z_{\alpha} Z_{\zeta} \zeta}} \mu^{-\epsilon / 2}, \\
& \bar{f}=-\frac{\frac{Z_{A} Z_{\tau \tau} Z_{\chi} \chi}{Z_{\zeta} \zeta}+Z_{A} Z_{\tau} Q}{\sqrt{-2 \alpha+\frac{Z_{\chi}^{2} \chi^{2}}{Z_{\alpha} Z_{\zeta}}}} \mu^{\epsilon / 2},
\end{aligned}
$$

in order to obtain a new expression for $\Gamma$ involving only terms linear in the sources. The renormalization factors $(Z$ factors) are calculable, see [47] and underlying references like $[45,70]$. In the $\overline{\mathrm{MS}}$ scheme and at one-loop, these $Z$ factors read in our current conventions as follows:

$$
\begin{aligned}
& Z_{A}=1+\frac{13}{6} \frac{N g^{2}}{16 \pi^{2}} \frac{2}{\epsilon}, \quad Z_{g}=1-\frac{11}{6} \frac{N g^{2}}{16 \pi^{2}} \frac{2}{\epsilon}, \\
& Z_{\varphi}=Z_{g}^{-1} Z_{A}^{-1 / 2}=1+\frac{3}{4} \frac{N g^{2}}{16 \pi^{2}} \frac{2}{\epsilon},
\end{aligned}
$$

$Z_{\zeta}=1-\frac{13}{6} \frac{N g^{2}}{16 \pi^{2}} \frac{2}{\epsilon}, \quad Z_{\alpha}=1+\frac{35}{12} \frac{N g^{2}}{16 \pi^{2}} \frac{2}{\epsilon}, \quad Z_{\chi}=1$,

$Z_{\gamma^{2}}=Z_{g}^{-1 / 2} Z_{A}^{-1 / 4}=1+\frac{3}{8} \frac{N g^{2}}{16 \pi^{2}} \frac{2}{\epsilon}$,

$Z_{\tau \tau}=1-\frac{35}{12} \frac{N g^{2}}{16 \pi^{2}} \frac{2}{\epsilon}, \quad Z_{\tau Q}=0$,

$Z_{Q Q}=Z_{\varphi}^{-1}=1-\frac{3}{2} \frac{N g^{2}}{16 \pi^{2} \epsilon}$.

Therefore, (18) can be rewritten as follows:

$$
\begin{aligned}
e^{-\Gamma(Q, \tau)}= & \int[\mathcal{D} \Phi]\left[\mathcal{D} \sigma_{1} \mathcal{D} \sigma_{2}^{\prime}\right] \exp \left[-S_{\mathrm{GZ}}\right. \\
& -\int d^{d} x\left(\frac{\sigma_{1}^{2}}{2 Z_{\zeta}}\left(1-\frac{\bar{b}^{2}}{\bar{e}^{2}} \frac{Z_{\alpha}}{Z_{\zeta}}\right)\right. \\
& -\frac{\sigma_{2}^{\prime 2}}{2 Z_{\alpha}}-\frac{\bar{b}}{\bar{e}} \frac{\sigma_{1} \sigma_{2}^{\prime}}{Z_{\zeta}} \\
& +\left(\frac{1}{2 Z_{\zeta}}\left(\bar{a}-\frac{\bar{f} \bar{b}}{\bar{e}}\right) \sigma_{1}-\frac{\bar{f}}{2 Z_{\alpha}} \sigma_{2}^{\prime}\right) A^{2} \\
& -\left(\frac{\bar{b} \bar{d}}{\bar{e}} \frac{1}{Z_{\zeta}} \sigma_{1}+\frac{\bar{d}}{Z_{\alpha}} \sigma_{2}^{\prime}\right) \bar{\varphi} \varphi \\
& +\frac{\bar{a}^{2}}{8 Z_{\zeta}}\left(A^{2}\right)^{2}-\frac{1}{2 Z_{\alpha}}\left(\frac{\bar{f}}{2} A^{2}+\bar{d} \bar{\varphi} \varphi\right)^{2} \\
& \left.\left.+\frac{\bar{c}}{Z_{\zeta}} \sigma_{1} \tau-\frac{\bar{e}}{Z_{\alpha}} \sigma_{2}^{\prime} Q\right)\right]
\end{aligned}
$$

where $\sigma_{2}^{\prime}$ is defined by

$\sigma_{2}^{\prime}=\sigma_{2}-\frac{\bar{b}}{\bar{e}} \frac{Z_{\alpha}}{Z_{\zeta}} \sigma_{1}$

In this expression, all LCO parameters, sources and fields are now finite, and infinities are only present in the $Z$ renormalization factors, whether explicitly written or present in the coefficients $\bar{a}$ to $\bar{f}$. At one loop, $\chi=0$ and $Z_{\tau Q}=0$ [47], which implies that $\bar{b}=\bar{f}=0$ and thus $\sigma_{2}^{\prime}=\sigma_{2}$.

In order to have an expression of the form $\frac{m^{2}}{2} A^{2}-M^{2} \bar{\varphi} \varphi$, we define the effective mass scales, $m^{2}$ and $M^{2}$, linked to $\langle A A\rangle$ and $\langle\bar{\varphi} \varphi\rangle$ respectively, by the classical (leading order in $g$ ) parts of the vacuum expectation values of the respective quadratic terms in the action (27), that is:

$$
\begin{aligned}
m^{2} & \left.\equiv\left(\frac{1}{Z_{\zeta}}\left(\bar{a}-\frac{\bar{f} \bar{b}}{\bar{e}}\right)\left\langle\sigma_{1}\right\rangle-\frac{\bar{f}}{Z_{\alpha}}\left\langle\sigma_{2}^{\prime}\right\rangle\right)\right|_{\text {leading }} \\
& =\left.\frac{1}{\sqrt{\zeta}}\right|_{\text {leading }}\left\langle\sigma_{1}\right\rangle=\sqrt{\frac{13 N g^{2}}{9\left(N^{2}-1\right)}}\left\langle\sigma_{1}\right\rangle
\end{aligned}
$$




$$
\begin{aligned}
M^{2} & \left.\equiv\left(\frac{\bar{b} \bar{d}}{\bar{e}} \frac{1}{Z_{\zeta}}\left\langle\sigma_{1}\right\rangle+\frac{\bar{d}}{Z_{\alpha}}\left\langle\sigma_{2}^{\prime}\right\rangle\right)\right|_{\text {leading }} \\
& =\left.\frac{1}{\sqrt{-2 \alpha}}\right|_{\text {leading }}\left\langle\sigma_{2}^{\prime}\right\rangle=\sqrt{\frac{35 N g^{2}}{48\left(N^{2}-1\right)^{2}}}\left\langle\sigma_{2}^{\prime}\right\rangle
\end{aligned}
$$

where the last equalities follow from $\alpha=\frac{\alpha_{0}}{g^{2}}=-\frac{24\left(N^{2}-1\right)^{2}}{35 \mathrm{Ng}^{2}}$ and $\zeta=\frac{\zeta_{0}}{g^{2}}=\frac{9\left(N^{2}-1\right)}{13 N g^{2}}[47]$.

Assuming the fields $\sigma_{1}$ and $\sigma_{2}^{\prime}$ develop nonzero vacuum expectation values, we can compute these by means of Jackiw's background field method [71]. We replace these fields by a classical vacuum expectation value and a fluctuating quantum part, $\sigma \rightarrow\langle\sigma\rangle+\sigma$, ignore terms linear in the fields as these drop out when working around extrema, and we integrate out all the fluctuations. With this decomposition of the (auxiliary) fields, the quadratic part of the action (including only those $Z$-factors that are necessary for a one-loop computation) becomes

$$
\begin{aligned}
& \int d^{d} x\left(\frac{1}{2} A_{\mu}^{a}\left(-\delta_{\mu \nu} \partial^{2}+\left(1-\frac{1}{\alpha_{g}}\right) \partial_{\mu} \partial_{\nu}\right) A_{\nu}^{a}\right. \\
& +\bar{c}^{a} \partial^{2} c^{a}+\bar{\varphi}_{\mu}^{a b} \partial^{2} \varphi_{\mu}^{a b}-\bar{\omega}_{\mu}^{a b} \partial^{2} \omega_{\mu}^{a b} \\
& -\gamma^{2} g f^{a b c} A_{\mu}^{a}\left(\varphi_{\mu}^{b c}+\bar{\varphi}_{\mu}^{b c}\right)-Z_{\gamma^{2}}^{2} d\left(N^{2}-1\right) \gamma^{4}+\frac{\sigma_{1}^{2}}{2 Z_{\zeta}} \\
& \left.-\frac{\sigma_{2}^{\prime 2}}{2 Z_{\alpha}}+\frac{m^{2}}{2} A^{2}-M^{2} \bar{\varphi} \varphi\right) .
\end{aligned}
$$

Using the definitions (29), in addition to

$$
\begin{aligned}
\bar{\varphi}_{\mu}^{a b} & =U_{\mu}^{a b}+i V_{\mu}^{a b}, \\
\varphi_{\mu}^{a b} & =U_{\mu}^{a b}-i V_{\mu}^{a b}, \\
P_{\mu \nu} & \equiv\left(-\partial^{2}+M^{2}\right) \delta_{\mu \nu}, \\
Q_{\mu \nu} & \equiv\left[\left(-\partial^{2}+m^{2}\right) \delta_{\mu \nu}+\left(1-\frac{1}{\alpha_{g}}\right) \partial_{\mu} \partial_{\nu}\right],
\end{aligned}
$$

this quadratic part can be rewritten as

$$
\begin{aligned}
& \int d^{d} x\left(-Z_{\gamma^{2}}^{2} d\left(N^{2}-1\right) \gamma^{4}\right. \\
& +\frac{9\left(N^{2}-1\right)}{13 N g^{2}} \frac{m^{4}}{2 Z_{\zeta}}-\frac{48\left(N^{2}-1\right)^{2}}{35 N g^{2}} \frac{M^{4}}{2 Z_{\alpha}} \\
& +\frac{1}{2} A_{\mu}^{a} Q_{\mu \nu} A_{\nu}^{a}+\bar{c}^{a} \partial^{2} c^{a} \\
& -U_{\mu}^{a b} P_{\mu \nu} U_{\nu}^{a b}-V_{\mu}^{a b} P_{\mu \nu} V_{\nu}^{a b} \\
& \left.-\bar{\omega}_{\mu}^{a b} \partial^{2} \omega_{\mu}^{a b}-2 \gamma^{2} g f^{a b c} A_{\mu}^{a} U_{\mu}^{b c}\right) .
\end{aligned}
$$

Since the ghost fields $c, \bar{c}, \omega, \bar{\omega}$ appear uncoupled to other fields, they can be immediately integrated out, giving just an overall factor. The real bosonic fields $U$ and $V$ can be integrated out next, leading to:

$$
\begin{aligned}
& \int[\mathcal{D} U][\mathcal{D} V] e^{-\int d^{d} x\left[-V_{\mu}^{a b} P_{\mu \nu} V_{\nu}^{a b}-U_{\mu}^{a b} P_{\mu \nu} U_{\nu}^{a b}-2 g \gamma^{2} f^{a b c} A_{\mu}^{a} U_{\mu}^{b c}\right]} \\
& =\frac{1}{\operatorname{det}\left(P_{\mu \nu} \delta^{a c} \delta^{b d}\right)} e^{-\int d^{d} x\left[N g^{2} \gamma^{4} A_{\mu}^{a} P_{\mu \nu}^{-1} \delta^{a b} A_{\nu}^{b}\right]}
\end{aligned}
$$

Introducing

$$
\begin{aligned}
& R_{\mu \nu} \equiv Q_{\mu \nu}+2 N g^{2} \gamma^{4} P_{\mu \nu}^{-1} \\
& \quad=\left[\left(-\partial^{2}+m^{2}+\frac{2 N \gamma^{4} g^{2}}{-\partial^{2}+M^{2}}\right) \delta_{\mu \nu}+\left(1-\frac{1}{\alpha}\right) \partial_{\mu} \partial_{\nu}\right],
\end{aligned}
$$

we now also integrate over the gluon field $A_{\mu}$. The quadratic part of the action containing $A_{\mu}$ is

$$
\int[\mathcal{D} A] e^{-\frac{1}{2} \int d^{d} x A_{\mu}^{a} R_{\mu \nu} A_{\nu}^{a}}=\frac{1}{\sqrt{\operatorname{det}\left(R_{\mu \nu} \delta^{a b}\right)}}
$$

As a result, the effective potential will $b^{2}$

$$
\begin{aligned}
\Gamma= & -Z_{\gamma^{2}}^{2} d\left(N^{2}-1\right) \gamma^{4}+\frac{9\left(N^{2}-1\right)}{13 N g^{2}} \frac{m^{4}}{2 Z_{\zeta}} \\
& -\frac{48\left(N^{2}-1\right)^{2}}{35 N g^{2}} \frac{M^{4}}{2 Z_{\alpha}}+\left(N^{2}-1\right)^{2} \operatorname{Tr} \ln P_{\mu \nu} \\
& +\frac{N^{2}-1}{2} \operatorname{Tr} \ln R_{\mu \nu} .
\end{aligned}
$$

The traces appearing in this expression are computed in the Appendix, see (A9). Also defining $\lambda^{4} \equiv 2 N g^{2} \gamma^{4}$, the one-loop renormalized effective potential of the GribovZwanziger theory, refined with the condensates $\left\langle A_{\mu}^{a} A_{\mu}^{a}\right\rangle$ and $\left\langle\bar{\varphi}_{\mu}^{a b} \varphi_{\mu}^{a b}\right\rangle$, reads:

$$
\begin{aligned}
& \Gamma\left(m^{2}, M^{2}, \lambda^{4}\right) \\
&=-\frac{2\left(N^{2}-1\right)}{N g^{2}} \lambda^{4}\left(1-\frac{3}{8} \frac{N g^{2}}{16 \pi^{2}}\right)+\frac{9\left(N^{2}-1\right)}{13 N g^{2}} \frac{m^{4}}{2} \\
&-\frac{48\left(N^{2}-1\right)^{2}}{35 N g^{2}} \frac{M^{4}}{2}+\frac{\left(N^{2}-1\right)^{2}}{8 \pi^{2}} M^{4}\left(-1+\ln \frac{M^{2}}{\bar{\mu}^{2}}\right) \\
&+\frac{3\left(N^{2}-1\right)}{64 \pi^{2}}\left(-\frac{5}{6}\left(m^{4}-2 \lambda^{4}\right)\right. \\
&+\frac{m^{4}+M^{4}-2 \lambda^{4}}{2} \ln \frac{m^{2} M^{2}+\lambda^{4}}{\bar{\mu}^{4}} \\
&-\left(m^{2}+M^{2}\right) \sqrt{4 \lambda^{4}-\left(m^{2}-M^{2}\right)^{2}} \\
&\left.\times \arctan \frac{\sqrt{4 \lambda^{4}-\left(m^{2}-M^{2}\right)^{2}}}{m^{2}+M^{2}}-M^{4} \ln \frac{M^{2}}{\bar{\mu}^{2}}\right) .
\end{aligned}
$$

In (37), $m^{2}$ and $M^{2}$ are proportional to the vacuum expectation values $\left\langle\sigma_{1}\right\rangle$ and $\left\langle\sigma_{2}^{\prime}\right\rangle$ of the auxiliary fields $\sigma_{1}$ and $\sigma_{2}^{\prime}$

\footnotetext{
${ }^{2}$ We have tacitly removed the global volume factor everywhere.
} 
introduced through the Hubbard-Stratonovich transformations (24), which may appear unphysical. However, acting with $\left.\frac{\delta}{\delta \tau}\right|_{\tau=Q=0}$ and $\left.\frac{\delta}{\delta Q}\right|_{\tau=Q=0}$ on (18) and (27) respectively, we get:

$$
\begin{aligned}
& \frac{1}{2} Z_{\tau} Z_{A}\left\langle A_{\mu}^{a} A_{\mu}^{a}\right\rangle=\sqrt{\zeta} \mu^{-\epsilon / 2}\left\langle\sigma_{1}\right\rangle, \\
& Z_{Q} Z_{\varphi}\left\langle\bar{\varphi}_{\mu}^{a c} \varphi_{\mu}^{a c}\right\rangle=-\sqrt{-2 \alpha} \mu^{-\epsilon / 2}\left\langle\sigma_{2}^{\prime}\right\rangle .
\end{aligned}
$$

The condensates $\left\langle\sigma_{1}\right\rangle$ and $\left\langle\sigma_{2}^{\prime}\right\rangle$, and so the mass scales $m^{2}$ and $M^{2}$ entering in $\Gamma$, are thus directly related to the more intuitive BRST invariant condensates $\left\langle A_{\mu}^{a} A_{\mu}^{a}\right\rangle_{\text {Landau }} \equiv\left\langle A_{\mu}^{h} A_{\mu}^{h}\right\rangle$ and $\left\langle\bar{\varphi}_{\mu}^{a c} \varphi_{\mu}^{a c}\right\rangle$ we were originally interested in, of which the LHS of (38) are the properly renormalized versions.

As expected, the condensation of the LCOs $A_{\mu}^{a} A_{\mu}^{a}$ and $\bar{\varphi}_{\mu}^{a c} \varphi_{\mu}^{a c}$ modifies the energy density $\Gamma$ of the theory. The three first terms in the first line form the classical part of the potential while the rest of $\Gamma$, proportional to $g^{2}$ when we consider the $g$-dependence of $m^{2}, M^{2}$ and $\lambda^{4}$, is the one-loop quantum correction.

\section{Gap equation and minimization}

We now proceed to find the physical state of the vacuum. We need to solve the gap equation (9) while simultaneously minimizing with respect to $m^{2}$ and $M^{2}$.

The minus sign in front of $M^{4}$ in the second classical term $^{3}$ obviously makes the classical potential unbounded from below and thus, unphysical. Our hope at this point was that the first order quantum correction could "turn" the potential, making it bounded from below - and possessing one or several minima - and thus physically meaningful at the quantum level. If it is the case, this would mean that this effective potential (37) would have the remarkable property of being a pure quantum object, having no physical classical limit when $\hbar \rightarrow 0$.

A very qualitative asymptotic study gives $\Gamma \sim M^{4} \ln M^{2}$ for $M^{2} \rightarrow+\infty$ - that is, the one-loop correction overtakes the classical term $-M^{4}$, as we hoped. Notice that this is qualitative at best, since taking field expectation values to infinity entails the presence of divergent logarithmic terms, making the efficacy of the perturbative computation of the effective action again questionable. This issue is always present and has a priori nothing to do with the sign of the classical term. A full-fledged renormalization group improvement of the effective action goes far beyond the scope of the current paper, in particular since we are dealing with a multiscale problem. How to best deal with large expectation values in such cases

\footnotetext{
3 This is related to the sign of $\alpha_{0}$, which is ultimately dictated by the sign choice in the unity (24b) we used.
}

is yet unsettled, see e.g. [72-75] for possible strategies, both old and new ones.

\subsection{Strategy to search for solutions}

To find the vacuum state of the theory, we need to solve the following gap equations:

$$
\frac{\partial \Gamma}{\partial M^{2}}=0, \quad \frac{\partial \Gamma}{\partial m^{2}}=0, \quad \frac{\partial \Gamma}{\partial \lambda^{4}}=0
$$

As it is not possible to solve this very nonlinear system of equations by hand, we need to work numerically. In this case, it is necessary to make a choice for the renormalization scale $\bar{\mu}$ and the coupling $g$ before it is possible to start hunting for solutions. These choices are subject to several conditions: as we are working in a semiclassical approximation, we should choose $g$ to be sufficiently small that we can trust the perturbative approximation. The renormalization group then requires that $\bar{\mu}$ be sufficiently large, for we have (at one loop in the $\overline{M S}$ scheme)

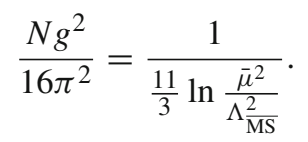

Furthermore, the scale $\bar{\mu}^{2}$ should be somehow "close" to the scales that appear in the logarithms (combinations of $\mathrm{m}^{2}$, $M^{2}$, and $\lambda^{2}$ ), lest the logarithms appearing in higher-order corrections be too big to warrant a first-order approximation. In addition, the solution should be stable under variation of $m^{2}$ and $M^{2}$, as these will take the value that minimizes the action. ${ }^{4}$ Finally, the existence of a nonzero solution for $\lambda$ is also a requirement, as otherwise the horizon condition would not be imposed, and the formalism would be again plagued by Gribov copies .

To investigate this last requirement, let us write down the gap equation for the Gribov parameter $\lambda$, and use the renormalization group equation (40) to eliminate the coupling $g$ in favor of $\bar{\mu}$ and $\Lambda_{\overline{\mathrm{MS}}}$ :

$x \operatorname{arccot} x=\frac{5}{6}-\frac{1}{2} \ln \frac{t}{\bar{\mu}^{4}}+\frac{44}{9} \ln \frac{\Lambda_{\overline{\mathrm{MS}}}^{2}}{\bar{\mu}^{2}}$,

where we used the shorthands

$x=\frac{m^{2}+M^{2}}{\sqrt{4 \lambda^{4}-\left(m^{2}-M^{2}\right)^{2}}}, \quad t=m^{2} M^{2}+\lambda^{4}$.

\footnotetext{
4 The value of $\lambda^{2}$ only needs to extremize the action, and indeed will normally maximize it. Although the latter might sound counterintuitive, it is actually a good sign. Indeed, we recall here that the original parameter $\gamma^{2}$ is the critical point coming from a saddle point evaluation $[30,56]$, so it better be corresponding to a maximum. The other parameters $m^{2}$ and $M^{2}$, however, need to be such that they minimize the action, for fixed $\gamma^{2}$. This means we need to verify, at the end, with the Hessian determinant criterion, that we have effectively found a minimum solution of the last 2 gap equations (39).
} 
In this Eq. (41), there is still one choice we have to make: the value of $\bar{\mu}$. To simplify the computation, we will follow a backward approach: we will choose a value ${ }^{5}$ for $x \operatorname{arccot} x$, which determines $x$ and thus $\lambda$ as a function of the as yet undetermined $m^{2}$ and $M^{2}$. Next, we solve (still by hand) the gap equation (41) for $\bar{\mu}$ as a function of $m^{2}$ and $M^{2}$. Putting these solutions into the gap equations for $m^{2}$ and $M^{2}$, we can solve numerically for these two mass parameters. Plugging the solution back into the expressions we found for $\lambda$ and $\bar{\mu}$, we can determine the numerical values for these parameters as well.

Once a numerical solution has been found, we have to inspect its characteristics to see whether the solution is acceptable. In the $\overline{\mathrm{MS}}$ scheme for $N=3$, it turns out that the effective coupling $N g^{2} / 16 \pi^{2}$ is quite large for any value of $x \operatorname{arccot} x$ we may choose in (41). The lowest value we obtained was $N g^{2} / 16 \pi^{2}=1.7$. Other choices yielded either higher values of the coupling constant, or nonsensical negative $g^{2}$ values, or a saddlepoint when varying $m^{2}$ and $M^{2}$.

As the difficulty to find satisfactory solutions may be due to $\overline{\mathrm{MS}}$ not being the most convenient subtraction scheme, we investigated other schemes. A scheme which is often used is the momentum subtraction (MOM) scheme, as it can also be easily implemented on a lattice. The relationship between this scheme and $\overline{\mathrm{MS}}$ is computed in detail in [76]. In our case, it turns out the first term in (37) is to be replaced by

$$
-\frac{2\left(N^{2}-1\right)}{N g^{2}} \lambda^{4}\left(1-\left(\frac{3}{8}-\frac{5.233}{N}\right) \frac{N g^{2}}{16 \pi^{2}}\right) \text {. }
$$

Applying the procedure outlined above for $\overline{\mathrm{MS}}$ still did not yield any satisfactory solutions, though.

\subsection{General subtraction scheme and lattice input}

In order to overcome these issues, we will "optimize" our one-loop effective action by considering it in a generic scheme. As is argued in [77], we actually only need to parameterize two renormalization factors to change from the $\overline{\mathrm{MS}}$ scheme to a general scheme since there are only two independent $Z$-factors in Landau gauge. In our case, it turns out to be most useful to consider $Z_{g}$ and $Z_{\gamma^{2}}$ as the independent $Z$-factors, and adapt the other $Z$-factors accordingly. We also take into account that the LCO parameters always appear in combinations like $Z_{\zeta} \zeta$, the latter being renormalization group invariants themselves, see also the comments in [68]. As a result, again only the first term in (37) is modified, becoming

$$
-\frac{2\left(N^{2}-1\right)}{N g^{2}} \lambda^{4}\left(1-\left(\frac{3}{8}-b_{0}\right) \frac{N g^{2}}{16 \pi^{2}}\right) .
$$

\footnotetext{
$\overline{5}$ This value can be any positive real number. If we choose a value larger than one, $x$ will be purely imaginary and $4 \lambda^{4}<\left(m^{2}-M^{2}\right)^{2}$.
}

where $b_{0}$ is a free parameter linked to the renormalization of the Gribov parameter $\gamma^{2}$, i.e. to the finite part in the infinite renormalization factor $Z_{\gamma^{2}}$. The other freedom of scheme, lingering in the coupling constant renormalization, is yet invisible at one-loop order. As such, we can keep using the $\overline{\mathrm{MS}}$ coupling.

With this general subtraction scheme, we again apply the steps outlined in the previous subsection to solve numerically for the effective mass scales $m^{2}$ and $M^{2}$ and the Gribov parameter $\gamma^{2}$, now as functions of the parameter $b_{0}$ in addition to the renormalization scale $\bar{\mu}$. Choosing the value of $b_{0}$ appropriately now does yield acceptable solutions. Now, however, we have too much freedom, and we need some extra criterion to fix $b_{0}$ again.

Applying the principle of minimal sensitivity [78] did not give anything useful: there was no optimal parameter choice. As such, we propose a different approach. The ultimate goal of this research program is to investigate what happens with the Gribov-Zwanziger theory at finite temperature, to investigate the response of the Green functions and their feedback on the deconfinement transition, if any, which can be investigated by including an appropriate temporal background [7982], which allows to access the vacuum expectation value of the Polyakov loop. An important first step in this direction is to pinpoint a desirable $T=0$ vacuum state to start from. As such, we will benefit from lattice studies, of both SU(2) and $\mathrm{SU}(3)$ gauge theories, that have investigated how well a propagator of the Gribov type can describe the lattice gluon propagator, see $[65,66]$. We will, however, not directly match our mass scales to the corresponding ones on the lattice, as this is a renormalization scheme and scale dependent operation. Instead we should use renormalization group invariant mass scales, which will be scale and scheme independent.

In the (R)GZ setting, there are two natural candidates, namely the set of complex conjugate poles of the gluon propagator (13). Next to being scale and scheme independent as pole masses, ${ }^{6}$ these quantities are even gauge parameter independent, thanks to the underlying BRST invariance, encoded in Nielsen identities $[83,84]$. Practically speaking, we determine the complex conjugate poles of our propagator (13) using the input of the one-loop effective potential, which depends on the 2 parameters $b_{0}$ and $\bar{\mu}$, and we determine the latter two values by matching our estimate of these gluon poles with those as estimated from the lattice data, [66] for $N=3$ and [65] for $N=2$.

Let us first discuss the $N=3$ case. From the data given at the bottom of page 358 of Ref. [66], righthand numbers, we can read off the denominator of the gluon propagator as $p^{4}+0.522 \mathrm{GeV}^{2} p^{2}+0.2845 \mathrm{GeV}^{4}$, from which the poles of the gluon propagator (which are our $x_{ \pm}$, see (A7)) are

\footnotetext{
6 The generalization of the standard lore that a pole mass has these properties has been extended to the (R)GZ theory as well, see [40].
} 

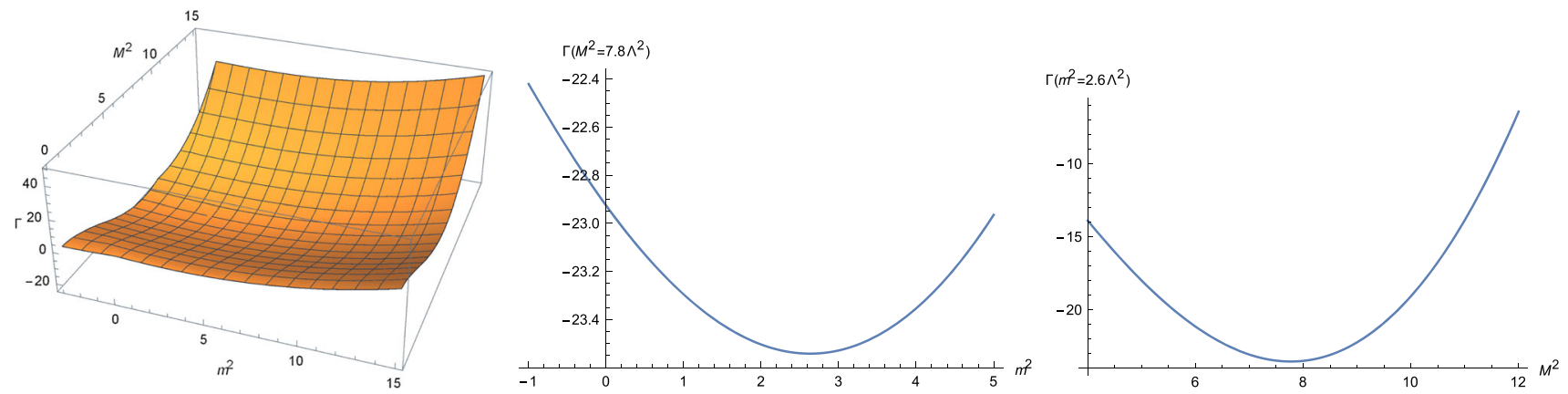

Fig. 1 The effective action (left) and 2 slices thereof illustrating the minimum (right) $\left(N=3\right.$ case, in units of $\left.\Lambda_{\overline{\mathrm{MS}}}\right)$

$$
-\left.p^{2}\right|_{\text {pole }}=(0.26 \pm i 0.47) \mathrm{GeV}^{2}=(5.2 \pm i 9.3) \Lambda \frac{2}{\mathrm{MS}}
$$

where we used that $\Lambda_{\overline{\mathrm{MS}}}=0.224 \mathrm{GeV}$ in $N=3$ pure YangMills $[85,86]$. A careful numerical analysis, following the above methodology, yields that for

$x \operatorname{arccot} x=0.82, \quad b_{0}=-3.42$

the equations allow for a solution with the gluon propagator pole at the right spot. In this solution we have

$$
\begin{aligned}
& \frac{g^{2} N}{16 \pi^{2}}=0.40, \quad \bar{\mu}=1.41 \Lambda \overline{\mathrm{MS}}=0.31 \mathrm{GeV}, \\
& \Gamma=-24 \Lambda \overline{\mathrm{MS}}^{4}=-0.059 \mathrm{GeV}^{4} \\
& \lambda^{4}=28 \Lambda \overline{\mathrm{MS}}^{4}=0.071 \mathrm{GeV}^{4} \\
& m^{2}=2.6 \Lambda \overline{\mathrm{MS}}^{2}=0.13 \mathrm{GeV}^{2} \\
& M^{2}=7.8 \Lambda \overline{\mathrm{MS}}^{2}=0.39 \mathrm{GeV}^{2}
\end{aligned}
$$

It turns out that the effective coupling constant is sufficiently small to attribute a qualitatively trustworthy meaning to our results. Furthermore we checked that the solution is a minimum under variation of $m^{2}$ and $M^{2}$ by computing the Hessian matrix at the minimum.

The main features of the above solution are captured in Fig. 1. It is instructive to notice that the vacuum energy is strictly negative, which shows that the non-perturbative vacuum in presence of the non-vanishing BRST invariant $d=2$ condensates is, at least up to one loop order, more stable than the "pure" GZ vacuum $\left(m^{2}=M^{2}=0\right)$, in which case it was already shown in [77] that the vacuum energy is always strictly positive, independent of the choice of massless renormalization scheme. In fact, at $M^{2}=m^{2}=0$, we have for any choice of scale or scheme that, at one-loop, $\frac{\partial \Gamma}{\partial m^{2}}<0$, $\frac{\partial \Gamma}{\partial M^{2}}<0$, so the pure GZ vacuum is indeed not stable.

To get an idea of the sensitivity to the choice of scale and scheme, let us also present the results where the optimum values (46) were increased with $25 \%$,

$$
\begin{aligned}
& \frac{g^{2} N}{16 \pi^{2}}=0.30, \quad \bar{\mu}=1.57 \Lambda \overline{\mathrm{MS}}=0.35 \mathrm{GeV} \\
& \Gamma=-65 \Lambda \overline{\mathrm{MS}}^{4}=-0.16 \mathrm{GeV}^{4} \\
& \lambda^{4}=27 \Lambda_{\overline{\mathrm{MS}}}^{4}=0.069 \mathrm{GeV}^{4} \\
& m^{2}=2.3 \Lambda_{\overline{\mathrm{MS}}}^{2}=0.11 \mathrm{GeV}^{2} \\
& M^{2}=12.74 \Lambda_{\overline{\mathrm{MS}}}^{2}=0.64 \mathrm{GeV}^{2}
\end{aligned}
$$

or decreased with $25 \%$,

$$
\begin{aligned}
& \frac{g^{2} N}{16 \pi^{2}}=0.66, \quad \bar{\mu}=1.23 \Lambda \overline{\mathrm{MS}}=0.27 \mathrm{GeV} \\
& \Gamma=-6 \Lambda_{\overline{\mathrm{MS}}}^{4}=-0.016 \mathrm{GeV}^{4} \\
& \lambda^{4}=45 \overline{\mathrm{MS}}^{4}=0.11 \mathrm{GeV}^{4} \\
& m^{2}=3.59 \Lambda_{\overline{\mathrm{MS}}}^{2}=0.18 \mathrm{GeV}^{2} \\
& M^{2}=4.35 \Lambda \overline{\mathrm{MS}}^{2}=0.22 \mathrm{GeV}^{2}
\end{aligned}
$$

For completeness, in Fig. 2, we display the dependence of $\Gamma$ in the region with solutions, this to appreciate the fact that there is no optimal solution in the sense of minimal sensitivity. This relatively strong dependence on the renormalization scale and scheme suggests that more stable results will, possibly, only be achieved within a full-blown higher loop study. This goes beyond the scope of the current paper. Two-loop computations in the (R)GZ context have not been established so far due to the large number of diagrams, not only caused by the extra vertices but also by, in particular, the several mixed propagators involving the gluon and extra GZ fields.

For SU(2), we can be more brief. We use the results of [65]. In Table IV of this paper, the poles of the propagator are given as

$$
-\left.p^{2}\right|_{\text {pole }}=(0.29 \pm i 0.66) \mathrm{GeV}^{2}=(2.6 \pm i 6.0) \Lambda_{\mathrm{MS}}^{2},
$$




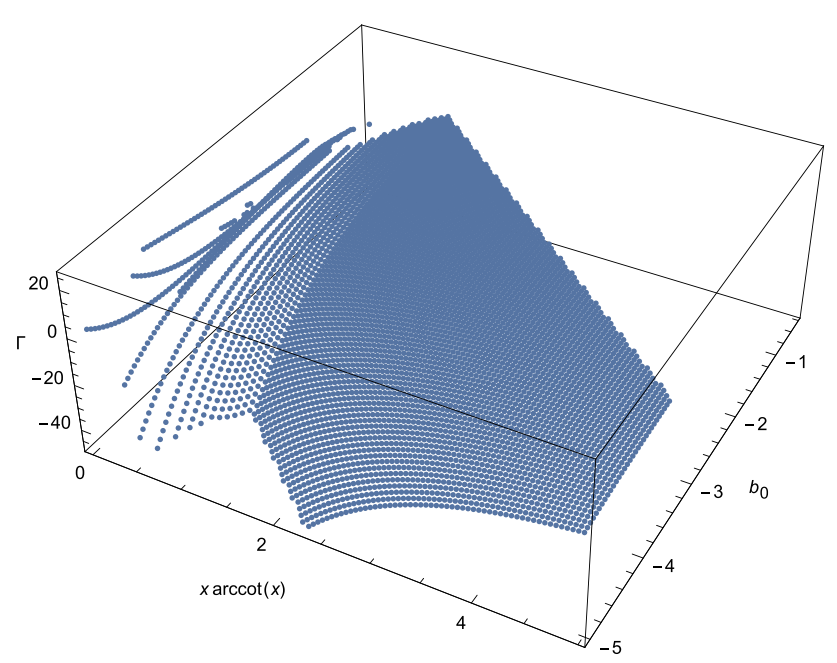

Fig. 2 Dependence of $\Gamma$ on the renormalization scale and scheme $\left(N=3\right.$ case, in units of $\left.\Lambda_{\overline{\mathrm{MS}}}\right)$

where we used that $\Lambda_{\overline{\mathrm{MS}}}=0.331 \mathrm{GeV}$ in $N=2$ pure YangMills [86,87]. We found that for

$x \operatorname{arccot} x=0.74, \quad b_{0}=-1.6$

the equations yielded a solution with the gluon propagator pole at the right spot. In this solution we have

$$
\begin{aligned}
& \frac{g^{2} N}{16 \pi^{2}}=1.24, \quad \bar{\mu}=1.12 \Lambda \overline{\mathrm{MS}}=0.37 \mathrm{GeV}, \\
& \Gamma=-0.38 \Lambda_{\overline{\mathrm{MS}}}^{4}=-0.0046 \mathrm{GeV}^{4} \\
& \lambda^{4}=9.1 \Lambda_{\overline{\mathrm{MS}}}^{4}=0.109 \mathrm{GeV}^{4} \\
& m^{2}=2.3 \Lambda_{\overline{\mathrm{MS}}}^{2}=0.25 \mathrm{GeV}^{2} \\
& M^{2}=2.9 \Lambda_{\overline{\mathrm{MS}}}^{2}=0.32 \mathrm{GeV}^{2} .
\end{aligned}
$$

It turns out that the effective coupling constant is again a bit too high to really trust the $S U(2)$ results; we notice that the SU(2) and SU(3) results are in the same ballpark, related to the fact of course the input pole masses were rather similar.

\section{Conclusion}

In this paper, we considered the recently introduced GribovZwanziger action that implements the restriction of the gauge degrees of path integration to a smaller subregion in a way consistent with the linear covariant gauge condition while also removing a large set of Gribov (gauge) copies. We have explicitly constructed the one-loop effective potential for two $d=2$ condensates, related to BRST invariant operators. The latter property allows to carry out their computation (as well as that of the effective potential) in a specific gauge. We opted for Landau gauge, in which case the computation simplifies most. As the considered operators are local but composite, care is needed in how to construct the effective potential, related to renormalization (group) issues. We relied on the LCO (local composite operator) formalism of $[45,47,48]$, which resolved all possible issues.

We computed the one-loop potential in a generic massless renormalization scheme, but were unable to pinpoint an optimal scheme, in the sense of minimal sensitivity. We therefore used lattice estimates for the set of complex conjugate poles of the gluon propagator, which are known to be renormalization group invariants. We then selected the (unique at the considered order) scheme in which the computed (tree level) complex conjugate poles match those lattice values. As such, we have identified a specific renormalization scheme to treat the divergences at zero temperature (the case considered here), upon which we can build in future work to discuss the interplay of condensates and Gribov gap equation with the temperature, with as ultimate goal to find out whether the GZ quantization can capture some essentials of the QCD thermodynamics and phase transitions, thereby putting on firmer footing preceding studies like [88-93].

The main result of this paper is the first explicit verification, albeit at one-loop order, that GZ dynamically transforms itself into RGZ thanks to the formation of nonperturbative $d=2$ mass scales, whilst respecting gauge and renormalization group invariance. At the level of the propagators in a generic linear covariant gauge, our results are at least qualitatively consistent with lattice or other functional methods output. This extends to vertices in the Landau gauge, for which many more results are available, see [94] and references therein.

Acknowledgements D. Vercauteren is grateful for the hospitality at KU Leuven, made possible through the Senior Fellowship SF/17/005. C. P. Felix is a Ph.D. student supported by the program Ciências sem Fronteiras-CNPq, 234112/2014-0. L. F. Palhares thanks the hospitality at KU Leuven-Kulak where this work was initiated; she is partially supported by the Brazilian agencies CNPq (Grants 454564/2014-7 and 305732/2016-1) and FAPERJ (Grant E-26/203.197/2017) and is part of the project INCT-FNA (Process no. 464898/2014-5). This study was financed in part by the Coordenação de Aperfeiçoamento de Pessoal de Nível Superior-Brasil (CAPES)—Financial Code 001 (M. N. F.). F. Rondeau is supported by Ecole Normale Supérieure Paris-Saclay, and thanks the hospitality of KU Leuven campus Kulak Kortrijk.

Data Availability Statement This manuscript has no associated data or the data will not be deposited. [Authors' comment: The numerical data on which our plots are based can be obtained from the authors upon request.]

Open Access This article is distributed under the terms of the Creative Commons Attribution 4.0 International License (http://creativecomm ons.org/licenses/by/4.0/), which permits unrestricted use, distribution, and reproduction in any medium, provided you give appropriate credit to the original author(s) and the source, provide a link to the Creative Commons license, and indicate if changes were made.

Funded by SCOAP ${ }^{3}$. 


\section{Appendix A: Technical computational details}

We compute the effective potential given in (36).

First of all there is the classical contribution

$$
\begin{aligned}
& -Z_{\gamma^{2}}^{2} d\left(N^{2}-1\right) \gamma^{4}+\frac{9\left(N^{2}-1\right)}{13 N g^{2}} \frac{m^{4}}{2 Z_{\zeta}} \\
& -\frac{48\left(N^{2}-1\right)^{2}}{35 N g^{2}} \frac{M^{4}}{2 Z_{\alpha}}
\end{aligned}
$$

Using the one-loop $Z$ factors given in (26), this evaluates to

$$
\begin{gathered}
-4\left(N^{2}-1\right) \gamma^{4}\left(1+\frac{3}{4} \frac{N g^{2}}{16 \pi^{2}} \frac{2}{\epsilon}-\frac{3}{8} \frac{N g^{2}}{16 \pi^{2}}\right) \\
+\frac{9\left(N^{2}-1\right)}{13 N g^{2}} \frac{m^{4}}{2}\left(1+\frac{13}{6} \frac{N g^{2}}{16 \pi^{2}} \frac{2}{\epsilon}\right) \\
-\frac{48\left(N^{2}-1\right)^{2}}{35 N g^{2}} \frac{M^{4}}{2}\left(1-\frac{35}{12} \frac{N g^{2}}{16 \pi^{2}} \frac{2}{\epsilon}\right) .
\end{gathered}
$$

To compute the logarithmic traces of $P_{\mu \nu}$ and $R_{\mu \nu}$, we use the following well-known expression

$$
\begin{aligned}
& \operatorname{Tr} \ln \left(-\partial^{2}+\Delta\right) \\
& \quad=-\frac{1}{(4 \pi)^{d / 2}} \Gamma\left(-\frac{d}{2}\right) \Delta^{d / 2} \\
& \quad=\frac{\Delta^{2}}{32 \pi^{2}}\left(-\frac{2}{\epsilon}-\frac{3}{2}+\ln \frac{\Delta}{\bar{\mu}^{2}}\right),
\end{aligned}
$$

where we used dimensional regularization $(d=4-\epsilon)$ and the $\overline{\mathrm{MS}}$ scheme. Using the fact that $\operatorname{tr} \delta_{\mu \nu}=d=4-\epsilon$, we immediately find that

$\operatorname{Tr} \ln P_{\mu \nu}=\frac{M^{4}}{8 \pi^{2}}\left(-\frac{2}{\epsilon}-1+\ln \frac{M^{2}}{\bar{\mu}^{2}}\right)$.

To compute the trace of the logarithm of $R_{\mu \nu}$, we first split the spectrum in one longitudinal polarization with eigenvalue $p^{2} / \alpha_{g}$, and $d-1$ transversal polarizations with eigenvalue

$p^{2}+m^{2}+\frac{2 N \gamma^{4} g^{2}}{p^{2}+M^{2}}$.

As the longitudinal polarizations contribute nothing but an irrelevant constant, we only need to compute

$\operatorname{Tr} \ln R_{\mu \nu}$

$$
\begin{aligned}
= & (d-1) \operatorname{Tr} \ln \left(p^{2}+m^{2}+\frac{2 N \gamma^{4} g^{2}}{p^{2}+M^{2}}\right) \\
= & (d-1) \operatorname{Tr} \ln \left(\left(p^{2}+m^{2}\right)\left(p^{2}+M^{2}\right)+2 N \gamma^{4} g^{2}\right) \\
& -(d-1) \operatorname{Tr} \ln \left(p^{2}+M^{2}\right) .
\end{aligned}
$$

Writing $2 N g^{2} \gamma^{4}=\lambda^{4}$ and introducing the solutions of the equation $x^{2}+\left(M^{2}+m^{2}\right) x+M^{2} m^{2}+\lambda^{4}=0$, namely

$x_{ \pm}=-\frac{1}{2}\left(m^{2}+M^{2} \pm \sqrt{\left(m^{2}-M^{2}\right)^{2}-4 \lambda^{4}}\right)$ this can be rewritten as

$$
\begin{aligned}
\operatorname{Tr} & \ln R_{\mu \nu} \\
= & (d-1) \operatorname{Tr} \ln \left(p^{2}-x_{+}\right) \\
& +(d-1) \operatorname{Tr} \ln \left(p^{2}-x_{-}\right) \\
& -(d-1) \operatorname{Tr} \ln \left(p^{2}+M^{2}\right) \\
= & \frac{3}{32 \pi^{2}}\left(m^{4}-2 \lambda^{4}\right)\left(-\frac{2}{\epsilon}-\frac{5}{6}\right) \\
& +\frac{3}{32 \pi^{2}}\left(x_{+}^{2} \ln \frac{-x_{+}}{\bar{\mu}^{2}}+x_{-}^{2} \ln \frac{-x_{-}}{\bar{\mu}^{2}}-M^{4} \ln \frac{M^{2}}{\bar{\mu}^{2}}\right) .
\end{aligned}
$$

Putting it all together, we find

$$
\begin{aligned}
\Gamma= & -4\left(N^{2}-1\right) \gamma^{4}\left(1-\frac{3}{8} \frac{N g^{2}}{16 \pi^{2}}\right) \\
& +\frac{9\left(N^{2}-1\right)}{13 N g^{2}} \frac{m^{4}}{2}-\frac{48\left(N^{2}-1\right)^{2}}{35 N g^{2}} \frac{M^{4}}{2} \\
& +\frac{\left(N^{2}-1\right)^{2}}{8 \pi^{2}} M^{4}\left(-1+\ln \frac{M^{2}}{\bar{\mu}^{2}}\right) \\
& +\frac{3\left(N^{2}-1\right)}{64 \pi^{2}}\left(-\frac{5}{6}\left(m^{4}-2 \lambda^{4}\right)+x_{+}^{2} \ln \frac{-x_{+}}{\bar{\mu}^{2}}\right. \\
& \left.+x_{-}^{2} \ln \frac{-x_{-}}{\bar{\mu}^{2}}-M^{4} \ln \frac{M^{2}}{\bar{\mu}^{2}}\right) .
\end{aligned}
$$

The full effective potential is thus finite in the limit $\epsilon \rightarrow 0$ at first order in $g^{2}$, a nontrivial result and a strong indication that the computation is consistent. One can also verify the invariance of $\Gamma$ under the renormalization group.

\section{References}

1. C.D. Roberts, A.G. Williams, Dyson-Schwinger equations and their application to hadronic physics. Prog. Part. Nucl. Phys. 33, 477-575 (1994)

2. R. Alkofer, L. von Smekal, The Infrared behavior of QCD Green's functions: confinement dynamical symmetry breaking, and hadrons as relativistic bound states. Phys. Rep. 353, 281 (2001)

3. D. Dudal, S.P. Sorella, N. Vandersickel, H. Verschelde, New features of the gluon and ghost propagator in the infrared region from the Gribov-Zwanziger approach. Phys. Rev. D 77, 071501 (2008)

4. D. Dudal, J.A. Gracey, S.P. Sorella, N. Vandersickel, H. Verschelde, A refinement of the Gribov-Zwanziger approach in the Landau gauge: infrared propagators in harmony with the lattice results. Phys. Rev. D 78, 065047 (2008)

5. A.C. Aguilar, D. Binosi, J. Papavassiliou, Gluon and ghost propagators in the Landau gauge: deriving lattice results from SchwingerDyson equations. Phys. Rev. D 78, 025010 (2008)

6. C.S. Fischer, A. Maas, J.M. Pawlowski, On the infrared behavior of Landau gauge Yang-Mills theory. Ann. Phys. 324, 2408-2437 (2009)

7. Ph Boucaud, J.P. Leroy, A. Le Yaouanc, J. Micheli, O. Pene, J. Rodriguez-Quintero, On the IR behaviour of the Landau-gauge ghost propagator. JHEP 06, 099 (2008) 
8. M. Tissier, N. Wschebor, Infrared propagators of Yang-Mills theory from perturbation theory. Phys. Rev. D 82, 101701 (2010)

9. N. Vandersickel, D. Zwanziger, The Gribov problem and QCD dynamics. Phys. Rep. 520, 175-251 (2012)

10. J. Serreau, M. Tissier, Lifting the Gribov ambiguity in Yang-Mills theories. Phys. Lett. B 712, 97-103 (2012)

11. A.K. Cyrol, M.Q. Huber, L. von Smekal, A Dyson-Schwinger study of the four-gluon vertex. Eur. Phys. J. C 75, 102 (2015)

12. F. Siringo, Second order gluon polarization for SU(N) theory in a linear covariant gauge. Phys. Rev. D 92(7), 074034 (2015)

13. M. Frasca, Quantum Yang-Mills field theory. Eur. Phys. J. Plus 132(1):38 (2017) [Erratum: Eur. Phys. J. Plus 132(5), 242 (2017)]

14. G. Comitini, F. Siringo, Variational study of mass generation and deconfinement in Yang-Mills theory. Phys. Rev. D 97(5), 056013 (2018)

15. M. Chaichian, M. Frasca, Condition for confinement in nonAbelian gauge theories. Phys. Lett. B 781, 33-39 (2018)

16. P.O. Bowman, U.M. Heller, D.B. Leinweber, M.B. Parappilly, A. Sternbeck, L. von Smekal, A.G. Williams, J.-B. Zhang, Scaling behavior and positivity violation of the gluon propagator in full QCD. Phys. Rev. D 76, 094505 (2007)

17. A. Cucchieri, T. Mendes, What's up with IR gluon and ghost propagators in Landau gauge? A puzzling answer from huge lattices. PoS LATTICE2007, 297 (2007)

18. A. Sternbeck, L. von Smekal, D.B. Leinweber, A.G. Williams, Comparing SU(2) to SU(3) gluodynamics on large lattices. PoS LATTICE2007, 340 (2007)

19. I.L. Bogolubsky, E.M. Ilgenfritz, M. Muller-Preussker, A. Sternbeck, The Landau gauge gluon and ghost propagators in 4D SU(3) gluodynamics in large lattice volumes. PoS LATTICE2007, 290 (2007)

20. A. Cucchieri, T. Mendes, Constraints on the IR behavior of the ghost propagator in Yang-Mills theories. Phys. Rev. D 78, 094503 (2008)

21. A. Maas, More on Gribov copies and propagators in Landau-gauge Yang-Mills theory. Phys. Rev. D 79, 014505 (2009)

22. O. Oliveira, P.J. Silva, The lattice Landau gauge gluon propagator: lattice spacing and volume dependence. Phys. Rev. D 86, 114513 (2012)

23. P. Bicudo, D. Binosi, N. Cardoso, O. Oliveira, P.J. Silva, Lattice gluon propagator in renormalizable $\xi$ gauges. Phys. Rev. D 92(11), $114514(2015)$

24. A. Cucchieri, T. Mendes, A.R. Taurines, Positivity violation for the lattice Landau gluon propagator. Phys. Rev. D 71, 051902 (2005)

25. D. Dudal, O. Oliveira, P.J. Silva, Källén-Lehmann spectroscopy for (un)physical degrees of freedom. Phys. Rev. D 89(1), 014010 (2014)

26. D. Dudal, O. Oliveira, M. Roelfs, P. Silva. Spectral representation of lattice gluon and ghost propagators at zero temperature. (2019). arXiv: 1901.05348

27. S. Strauss, C.S. Fischer, C. Kellermann, Analytic structure of the Landau gauge gluon propagator. Phys. Rev. Lett. 109, 252001 (2012)

28. A.K. Cyrol, J.M. Pawlowski, A. Rothkopf, N. Wink, Reconstructing the gluon. SciPost Phys. 5(6), 065 (2018)

29. D. Binosi, R.-A. Tripolt. Spectral functions of confined particles. (2019). arXiv:1904.08172

30. V.N. Gribov, Quantization of non Abelian gauge theories. Nucl. Phys. B 139, 1 (1978)

31. I.M. Singer, Some remarks on the Gribov ambiguity. Commun. Math. Phys. 60, 7-12 (1978)

32. D. Zwanziger, Local and renormalizable action from the Gribov horizon. Nucl. Phys. B 323, 513-544 (1989)

33. D. Zwanziger, Renormalizability of the critical limit of lattice gauge theory by BRS invariance. Nucl. Phys. B 399, 477-513 (1993)
34. C. Becchi, A. Rouet, R. Stora, The Abelian Higgs-Kibble model. Unitarity of the S operator. Phys. Lett. 52B, 344-346 (1974)

35. C. Becchi, A. Rouet, R. Stora, Renormalization of Gauge theories. Ann. Phys. 98, 287-321 (1976)

36. I.V. Tyutin. Gauge Invariance in Field Theory and Statistical Physics in Operator Formalism. P.N. Lebedev Physical Institute, No. 39 (1975)

37. M.A.L. Capri, D. Dudal, D. Fiorentini, M.S. Guimarães, I.F. Justo, A.D. Pereira, B.W. Mintz, L.F. Palhares, R.F. Sobreiro, S.P. Sorella, Exact nilpotent nonperturbative BRST symmetry for the GribovZwanziger action in the linear covariant gauge. Phys. Rev. D 92(4), 045039 (2015)

38. M.A.L. Capri, D. Fiorentini, M.S. Guimarães, B.W. Mintz, L.F. Palhares, S.P. Sorella, D. Dudal, I.F. Justo, A.D. Pereira, R.F. Sobreiro, More on the nonperturbative Gribov-Zwanziger quantization of linear covariant gauges. Phys. Rev. D 93(6), 065019 (2016)

39. M.A.L. Capri, D. Dudal, D. Fiorentini, M.S. Guimarães, I.F. Justo, A.D. Pereira, B.W. Mintz, L.F. Palhares, R.F. Sobreiro, S.P. Sorella, Local and BRST-invariant Yang-Mills theory within the Gribov horizon. Phys. Rev. D 94(2), 025035 (2016)

40. M.A.L. Capri, D. Dudal, A.D. Pereira, D. Fiorentini, M.S. Guimarães, B.W. Mintz, L.F. Palhares, S.P. Sorella, Nonperturbative aspects of Euclidean Yang-Mills theories in linear covariant gauges: Nielsen identities and a BRST-invariant two-point correlation function. Phys. Rev. D 95(4), 045011 (2017)

41. T. Kugo, I. Ojima, Local covariant operator formalism of nonabelian gauge theories and quark confinement problem. Prog. Theor. Phys. Suppl. 66, 1-130 (1979)

42. F.V. Gubarev, L. Stodolsky, V.I. Zakharov, On the significance of the vector potential squared. Phys. Rev. Lett. 86, 2220-2222 (2001)

43. F.V. Gubarev, V.I. Zakharov, On the emerging phenomenology of $<A^{2}>$. Phys. Lett. B 501, 28-36 (2001)

44. Ph Boucaud, A. Le Yaouanc, J.P. Leroy, J. Micheli, O. Pene, J. Rodriguez-Quintero, Testing Landau gauge OPE on the lattice with a $<A^{2}>$ condensate. Phys. Rev. D 63, 114003 (2001)

45. H. Verschelde, K. Knecht, K. Van Acoleyen, M. Vanderkelen, The non-perturbative groundstate of QCD and the local composite operator $A_{\mu}^{2}$. Phys. Lett. B 516, 307-313 (2001)

46. K.-I. Kondo, Vacuum condensate of mass dimension 2 as the origin of mass gap and quark confinement. Phys. Lett. B 514, 335-345 (2001)

47. D. Dudal, S.P. Sorella, N. Vandersickel, The dynamical origin of the refinement of the Gribov-Zwanziger theory. Phys. Rev. D 84, 065039 (2011)

48. H. Verschelde, Perturbative calculation of nonperturbative effects in quantum field theory. Phys. Lett. B 351, 242-248 (1995)

49. D. Dudal, H. Verschelde, S.P. Sorella, The anomalous dimension of the composite operator $A^{2}$ in the Landau gauge. Phys. Lett. B 555, 126-131 (2003)

50. M.A.L. Capri, D. Fiorentini, A.D. Pereira, R.F. Sobreiro, S.P. Sorella, R.C. Terin, Aspects of the refined Gribov-Zwanziger action in linear covariant gauges. Annals Phys. 376, 40-62 (2017)

51. G. Dell'Antonio, D. Zwanziger, Ellipsoidal bound on the Gribov horizon contradicts the perturbative renormalization group. Nucl. Phys. B 326, 333-350 (1989)

52. P. van Baal, More (thoughts on) Gribov copies. Nucl. Phys. B 369, 259-275 (1992)

53. M. Lavelle, D. McMullan, Constituent quarks from QCD. Phys. Rep. 279, 1-65 (1997)

54. M.A.L. Capri, D. Fiorentini, M.S. Guimarães, B.W. Mintz, L.F. Palhares, S.P. Sorella, Local and renormalizable framework for the gauge-invariant operator $A_{\mathrm{min}}^{2}$ in Euclidean Yang-Mills theories in linear covariant gauges. Phys. Rev. D 94(6), 065009 (2016)

55. G. Dell'Antonio, D. Zwanziger, Every gauge orbit passes inside the Gribov horizon. Commun. Math. Phys. 138, 291-299 (1991) 
56. M.A.L. Capri, D. Dudal, M.S. Guimaraes, L.F. Palhares, S.P. Sorella, An all-order proof of the equivalence between Gribov's no-pole and Zwanziger's horizon conditions. Phys. Lett. B 719, 448-453 (2013)

57. M.A.L. Capri, D.M. van Egmond, G. Peruzzo, M.S. Guimaraes, O. Holanda, S.P. Sorella, R.C. Terin, H.C. Toledo, On a renormalizable class of gauge fixings for the gauge invariant operator $A_{\min }^{2}$. Ann. Phys. 390, 214-235 (2018)

58. M.A.L. Capri, D. Fiorentini, A.D. Pereira, S.P. Sorella, Renormalizability of the refined Gribov-Zwanziger action in linear covariant gauges. Phys. Rev. D 96(5), 054022 (2017)

59. M.A.L. Capri, D. Fiorentini, S.P. Sorella, Gribov horizon and nonperturbative BRST symmetry in the maximal Abelian gauge. Phys. Lett. B 751, 262-271 (2015)

60. M.A.L. Capri, D. Fiorentini, A.D. Pereira, S.P. Sorella, A nonperturbative study of matter field propagators in Euclidean YangMills theory in linear covariant, Curci-Ferrari and maximal Abelian gauges. Eur. Phys. J. C 77(8), 546 (2017)

61. A. Cucchieri, T. Mendes, E.M.S. Santos, Covariant gauge on the lattice: a new implementation. Phys. Rev. Lett. 103, 141602 (2009)

62. M.Q. Huber, Gluon and ghost propagators in linear covariant gauges. Phys. Rev. D 91(8), 085018 (2015)

63. A.C. Aguilar, D. Binosi, J. Papavassiliou, Yang-Mills two-point functions in linear covariant gauges. Phys. Rev. D 91(8), 085014 (2015)

64. D. Dudal, O. Oliveira, N. Vandersickel, Indirect lattice evidence for the refined Gribov-Zwanziger formalism and the gluon condensate $\left\langle A^{2}\right\rangle$ in the Landau gauge. Phys. Rev. D 81, 074505 (2010)

65. A. Cucchieri, D. Dudal, T. Mendes, N. Vandersickel, Modeling the gluon propagator in Landau gauge: lattice estimates of pole masses and dimension-two condensates. Phys. Rev. D 85, 094513 (2012)

66. D. Dudal, O. Oliveira, P.J. Silva, High precision statistical Landau gauge lattice gluon propagator computation vs. the GribovZwanziger approach. Ann. Phys. 397, 351-364 (2018)

67. Y. Hayashi, K.-I. Kondo, Complex poles and spectral function of Yang-Mills theory. Phys. Rev. D 99(7), 074001 (2019)

68. K. Knecht, H. Verschelde, A new start for local composite operators. Phys. Rev. D 64, 085006 (2001)

69. V.E.R. Lemes, M.S. Sarandy, S.P. Sorella, Ghost condensates in Yang-Mills theories in the Landau gauge. Ann. Phys. 308, 1-17 (2003)

70. J.A. Gracey, Three loop MS-bar renormalization of the CurciFerrari model and the dimension two BRST invariant composite operator in QCD. Phys. Lett. B 552, 101-110 (2003)

71. R. Jackiw, Functional evaluation of the effective potential. Phys. Rev. D 9, 1686 (1974)

72. M.B. Einhorn, D.R.T. Jones, A new renormalization group approach to multiscale problems. Nucl. Phys. B 230, 261-272 (1984)

73. M. Bando, T. Kugo, N. Maekawa, H. Nakano, Improving the effective potential: multimass scale case. Prog. Theor. Phys. 90, 405-418 (1993)

74. C. Ford, D.R.T. Jones, P.W. Stephenson, M.B. Einhorn, The effective potential and the renormalization group. Nucl. Phys. B 395, 17-34 (1993)

75. L. Chataignier, T. Prokopec, M.G. Schmidt, B. Swiezewska, Single-scale renormalisation group improvement of multi-scale effective potentials. JHEP 03, 014 (2018)
76. J.A. Gracey, Two loop QCD vertices at the symmetric point. Phys. Rev. D 84, 085011 (2011)

77. D. Dudal, R.F. Sobreiro, S.P. Sorella, H. Verschelde, The Gribov parameter and the dimension two gluon condensate in Euclidean Yang-Mills theories in the Landau gauge. Phys. Rev. D 72, 014016 (2005)

78. P.M. Stevenson, Optimized perturbation theory. Phys. Rev. D 23, $2916(1981)$

79. F. Marhauser, J.M. Pawlowski, Confinement in Polyakov gauge. (2018). arXiv:0812.1144 [hep-ph]

80. J. Braun, H. Gies, J.M. Pawlowski, Quark confinement from color confinement. Phys. Lett. B 684, 262-267 (2010)

81. H. Reinhardt, J. Heffner, Effective potential of the confinement order parameter in the Hamiltonian approach. Phys. Rev. D 88, 045024 (2013)

82. U. Reinosa, J. Serreau, M. Tissier, N. Wschebor, Deconfinement transition in $\mathrm{SU}(N)$ theories from perturbation theory. Phys. Lett. B 742, 61-68 (2015)

83. N.K. Nielsen, On the gauge dependence of spontaneous symmetry breaking in gauge theories. Nucl. Phys. B 101, 173-188 (1975)

84. O. Piguet, K. Sibold, Gauge independence in ordinary Yang-Mills theories. Nucl. Phys. B 253, 517-540 (1985)

85. Ph Boucaud, F. De Soto, J.P. Leroy, A. Le Yaouanc, J. Micheli, O. Pene, J. Rodriguez-Quintero, Ghost-gluon running coupling, power corrections and the determination of $\Lambda_{\overline{\mathrm{MS}}}$. Phys. Rev. D 79, $014508(2009)$

86. D. Dudal, C.P. Felix, M.S. Guimarães, S.P. Sorella, Accessing the topological susceptibility via the Gribov horizon. Phys. Rev. D 96(7), 074036 (2017)

87. B. Lucini, G. Moraitis, The running of the coupling in $\mathrm{SU}(\mathrm{N})$ pure gauge theories. Phys. Lett. B 668, 226-232 (2008)

88. K. Fukushima, K. Kashiwa, Polyakov loop and QCD thermodynamics from the gluon and ghost propagators. Phys. Lett. B 723, 360-364 (2013)

89. K. Fukushima, N. Su, Stabilizing perturbative Yang-Mills thermodynamics with Gribov quantization. Phys. Rev. D 88, 076008 (2013)

90. F.E. Canfora, D. Dudal, I.F. Justo, P. Pais, L. Rosa, D. Vercauteren, Effect of the Gribov horizon on the Polyakov loop and vice versa. Eur. Phys. J. C 75(7), 326 (2015)

91. F. Canfora, D. Hidalgo, P. Pais. The Gribov problem in presence of background field for SU(2) Yang-Mills theory. Phys. Lett. B 763, 94-101 (2016) [Erratum: Phys. Lett. B 772, 880 (2017)]

92. D. Dudal, D. Vercauteren, Gauge copies in the Landau-DeWitt gauge: a background invariant restriction. Phys. Lett. B 779, 275$282(2018)$

93. D. Kroff, U. Reinosa, Gribov-Zwanziger type model action invariant under background gauge transformations. Phys. Rev. D 98(3), 034029 (2018)

94. B.W. Mintz, L.F. Palhares, S.P. Sorella, A.D. Pereira, Ghost-gluon vertex in the presence of the Gribov horizon. Phys. Rev. D 97(3), 034020 (2018) 